\title{
Instantaneous Signal and Self-Interference Power of MIMO Eigenmode Transmission with Feedback Time Delay
}

\author{
Ping-Heng Kuo*, Peter J. Smith*, Lee M. Garth* and Mansoor Shafi ${ }^{\dagger}$ \\ * Department of Electrical and Computer Engineering, \\ University of Canterbury, \\ Christchurch, New Zealand, \\ \{phk15,p.smith, 1.garth\}@elec.canterbury.ac.nz \\ $\dagger$ Telecom New Zealand Limited, \\ Wellington, New Zealand, \\ Mansoor.Shafi@telecom.co.nz
}

\begin{abstract}
Singular Value Decomposition (SVD) techniques are well-known for providing an elegant transceiver architecture, that can realize the intrinsic spatial links of a MIMO channel. Such a scheme is also referred to as eigen-beamforming or eigenmode transmission. The system performance is, however, closely related to the quality of the channel information at both ends of the link. Hence, we focus on the effect of feedback time delay, which causes the transmitter to use outdated channel information in timevarying fading channels. In this paper, we derive an analytical expression for the instantaneous SINR of eigenmode transmission with a feedback time delay $\tau$. Furthermore, this expression implies a novel metric that gauges the system performance sensitivity to time-variations of the steering vectors (eigenvectors of the channel correlation matrix) at the transmitter.
\end{abstract}

\section{INTRODUCTION}

In the capacity analysis of Multiple-Input Multiple-Output (MIMO) systems [1], the existence of decoupled scalar channels (eigenmodes) has been shown. This has inspired the use of singular value decomposition (SVD)-based MIMO transceiver architectures [2][3]. Since multiple independent data streams can be transmitted simultaneously through these uncoupled eigenmodes, the overall data rate can be improved significantly [4]. Nevertheless, this kind of system requires the transmitter to obtain channel state information (CSI) from the receiver with the aid of a feedback link.

In practice, neither end of the link acquires perfect CSI. There are two main impairments to the CSI accuracy: channel estimation error and feedback time delay [5]. In general, with imperfect CSI, the steering matrices (or weight matrices) at both ends are not able to completely decouple the eigenmodes. In this situation, the SVD transmission will experience additional self-interference. The mean capacity of such systems was approximated in [6] assuming an extremely large or small channel estimation error, but the effect of feedback delay was not considered. The influence of imperfect channel knowledge on the probability of error has been studied in [7] by means of simulation. In [8], a thorough analysis of this issue was undertaken, but it was assumed that the interference is spread evenly over all eigenmodes.

The goal of the research in this paper is to explicitly characterize the loss in signal power and the introduction of interference, due to the mismatch between the true and estimated steering matrices. To the best of our knowledge, no papers in the literature have addressed this problem before. For the sake of simplicity, we concentrate on the effects of feedback delay in this paper by assuming that the receiver tracks the channel perfectly (that is, channel estimation error is excluded, as in [9]); however, the analysis can be extended to cases with channel estimation error using the same principle, at the expense of more cumbersome calculations.

We analytically derive the instantaneous signal and interference power for eigenmode transmission with feedback delay in this paper. Hence, we can approximate the instantaneous signal to interference noise ratio (SINR). Based on these expressions, an eigenvalue dependent metric, which gauges the system performance sensitivity to time-variations of the steering matrices, is identified and proposed. The instantaneous SINR contains a power loss factor and an interference factor which are shown to be equal. This common factor is proportional to the square of the feedback delay. The proportionality constant is independent of the feedback delay, and its behavior is dependent on the radio channel. In particular, the proportionality constant contains terms which are inversely proportional to the difference between eigenvalues. Therefore, at times when this difference is small, there is a corresponding increase in the power loss and the interference. Similar observations have been made in [10], [11] and [12], where it was noted that a small variation in the channel can result in major shifts in the steering matrices, especially when two singular values (and therefore eigenvalues) are of a similar magnitue.

Hence, we have identified the effects of feedback delay in terms of a "background effect" proportional to the squared delay which is multiplied by a channel factor which measures the sensitivity of the channel to the delay. This leads to the interesting conclusion that any feedback delay, however small, can result in a degraded SINR if the channel is in a "sensitive" state.

The rest of the paper is organized as follows. In Sec. II, the system model is elaborated, and the SINR expression is 
derived in Sec. III. The novel channel metric is also discussed in Sec. III. Section IV presents our simulation results, and conclusions are given in Sec. V.

\section{MIMO SYSTEM MODEL}

Consider a $\left(N_{T}, N_{R}\right)$ MIMO system with $N_{T}$ transmit and $N_{R}$ receive antennas. We assume that the channel matrix $\boldsymbol{H}$ is an $N_{R} \times N_{T}$ matrix containing i.i.d. complex Gaussian entries with zero mean and unit magnitude variance (Rayleigh fading). We also denote $m=\min \left(N_{R}, N_{T}\right)$. Under the assumption of flat fading, the overall input-output relationship is simply

$$
\boldsymbol{y}=\boldsymbol{H} \boldsymbol{x}+\boldsymbol{n}
$$

where $\boldsymbol{x}, \boldsymbol{y}$ and $\boldsymbol{n}$ are transmitted data, received data and Gaussian noise, respectively. The noise vector is assumed to contain i.i.d. elements with zero mean and magnitude variance, $\sigma^{2}$. Applying the SVD, the channel matrix $\boldsymbol{H}$ can be decomposed as

$$
\boldsymbol{H}=\boldsymbol{U} \boldsymbol{D} \boldsymbol{V}^{\dagger}
$$

where $\boldsymbol{D}$ is a $N_{R} \times N_{T}$ matrix, containing $m$ non-negative elements on the diagonal, which are the singular values of $\boldsymbol{H}$. $\boldsymbol{U}$ and $\boldsymbol{V}$ are $N_{R} \times N_{R}$ and $N_{T} \times N_{T}$ unitary matrices, respectively. The overall input-output relationship is therefore

$$
\boldsymbol{y}=\boldsymbol{U} \boldsymbol{D} \boldsymbol{V}^{\dagger} \boldsymbol{x}+\boldsymbol{n} .
$$

Moreover, the columns of $\boldsymbol{U}$ are eigenvectors of $\boldsymbol{H} \boldsymbol{H}^{\dagger}$ and the columns of $\boldsymbol{V}$ are eigenvectors of $\boldsymbol{H}^{\dagger} \boldsymbol{H}$. If both the transmitter and receiver have perfect CSI, $\boldsymbol{U}$ and $\boldsymbol{V}$ can be computed, so one may simply transform the channel into a bank of $m$ scalar links by defining: $\widetilde{\boldsymbol{x}}=\boldsymbol{V}^{\dagger} \boldsymbol{x}, \widetilde{\boldsymbol{y}}=\boldsymbol{U}^{\dagger} \boldsymbol{y}$, and $\widetilde{\boldsymbol{n}}=\boldsymbol{U}^{\dagger} \boldsymbol{n}$. Hence, (3) can be re-written in the new equivalent form:

$$
\widetilde{\boldsymbol{y}}=\boldsymbol{D} \widetilde{\boldsymbol{x}}+\widetilde{\boldsymbol{n}}
$$

We denote the eigenvalues of $\boldsymbol{H} \boldsymbol{H}^{\dagger}$ (for $N_{R} \leq N_{T}$ ) or $\boldsymbol{H}^{\dagger} \boldsymbol{H}$ (for $N_{T}<N_{R}$ ), by $\lambda_{1} \geq \ldots \geq \lambda_{m}$. These eigenvalues are the power gains of the equivalent scalar excitations in $D$, i.e. the eigenmodes. Hence, the SVD-based MIMO architecture pre-filters $\boldsymbol{x}$ by $\boldsymbol{V}$ and post-filters $\boldsymbol{y}$ by $\boldsymbol{U}^{\dagger}$ to extract the eigenmodes for parallel communications. We term $\boldsymbol{V}$ and $\boldsymbol{U}$ steering matrices in the rest of the paper.

To study the effect of feedback delay we define the length of feedback delay to be $\tau$. We also assume that the temporal behavior of the channel coefficients is governed by the Jakes process with an auto-correlation function (ACF) $\rho(t)=J_{0}\left(2 \pi f_{D} t\right)$, where $J_{0}(\cdot)$ represents the zeroth order modified Bessel function, and $f_{D}$ is the Doppler frequency. We further define $\rho=\rho(\tau)$ to be ACF at lag $\tau$ which controls the change in the channel during the feedback delay. Note that the results in this paper can be extended to arbitary types of ACF. We now assume perfect CSI at the receiver and outdated channel information, delayed by $\tau$, at the transmitter. Thus, with the addition of a time index, (4) becomes

$$
\widehat{\boldsymbol{y}}(t)=\boldsymbol{D}(t) \boldsymbol{V}^{\dagger}(t) \boldsymbol{V}(t-\tau) \boldsymbol{x}(t)+\widetilde{\boldsymbol{n}}(t) .
$$

Clearly, the system cannot exactly diagonalize the channel due to the mismatch between $\boldsymbol{V}(t)$ and $\boldsymbol{V}(t-\tau)$. Hence the diagonal $\boldsymbol{D}$ in (4) is replaced by $\boldsymbol{D}(t) \boldsymbol{V}^{\dagger}(t) \boldsymbol{V}(t-\tau)$. In Sec. III we show that this has the effect of reducing the signal power and creating self-interference.

\section{Signal AND INTERFERENCE POWER CHARACTERIZATIONS}

In the preceding section, the effects of feedback delay on MIMO-SVD systems were explained. There are two main effects: the loss of signal power and the introduction of interference in the eigenmode transmissions. The goal of this section is to analytically approximate the instantaneous power of both components in any particular eigenmode transmission. To do this, it is necessary to use some results on Brownian matrices [13]. We develop these now.

\section{A. Bru's Theorem}

Consider a matrix $\boldsymbol{X}$, the entries of which are i.i.d. real Brownian motions. Bru [13] has derived a stochastic differential equation (SDE) for the elements of the eigenvectors of $\boldsymbol{X}^{T}(t) \boldsymbol{X}(t)$. Defining $\widetilde{\boldsymbol{V}}(t)=\left[\widetilde{v}_{i j}(t)\right]$ as the matrix of eigenvectors with $\widetilde{v}_{i j}(t)$ being the $i^{t h}$ element of the $j^{t h}$ eigenvector of $\boldsymbol{X}^{T}(t) \boldsymbol{X}(t)$, we have [13]:

$$
\begin{aligned}
d \widetilde{v}_{i j}(t)= & \sum_{k \neq j} \widetilde{v}_{i k}(t) \sqrt{\frac{w_{k}(t)+w_{j}(t)}{\left[w_{k}(t)-w_{j}(t)\right]^{2}}} d B_{k j}(t) \\
& -\frac{1}{2} \widetilde{v}_{i j}(t) \sum_{k \neq j} \frac{w_{k}(t)+w_{j}(t)}{\left[w_{k}(t)-w_{j}(t)\right]^{2}} d t
\end{aligned}
$$

where $B_{k j}(t)$ are independent Brownian motions and $w_{i}(t)$ are the eigenvalues of $\boldsymbol{X}^{T}(t) \boldsymbol{X}(t)$.

\section{B. A Modified SDE}

In this paper, we are interested in the matrix $\boldsymbol{V}(t)$ containing the eigenvectors of $\boldsymbol{H}^{\dagger}(t) \boldsymbol{H}(t)$. The elements of $\boldsymbol{H}(t)$ are complex Gaussians with unit variances, so the SDE (6) must be modified to accommodate our requirements. Following [14], the drift (the term containing $d t$ ) in the SDE for Wishart matrix eigenvalues is doubled for the complex Wishart case. This is also applicable to the eigenvector SDE, so the factor of $\frac{1}{2}$ in (6) is removed. Now, following the same arguments as in [15], the Brownian entries in $\boldsymbol{X}(t)$ have to be standardized. We replace $w_{i}$ with $2 t \lambda_{i}$, and look at the particular time point $t=$ $\tau\left[J_{0}\left(2 \pi f_{D} \tau\right)^{-2}-1\right]^{-1}$ as in [15], which in turn gives $\tau / t \approx$ $2 \pi^{2} f_{D}{ }^{2} \tau^{2}$. After some algebra, the Euler approximation to the modified SDE is given by

$$
\begin{aligned}
\Delta v_{i j}(t)= & \sqrt{2} \pi f_{D} \tau \sum_{k \neq j} v_{i k}(t-\tau) \sqrt{\frac{\lambda_{k}(t-\tau)+\lambda_{j}(t-\tau)}{\left[\lambda_{k}(t-\tau)-\lambda_{j}(t-\tau)\right]^{2}}} Z_{k j} \\
& -\pi^{2} f_{D}{ }^{2} \tau^{2} v_{i j}(t-\tau) \sum_{k \neq j} \frac{\lambda_{k}(t-\tau)+\lambda_{j}(t-\tau)}{\left[\lambda_{k}(t-\tau)-\lambda_{j}(t-\tau)\right]^{2}}
\end{aligned}
$$

where $\Delta v_{i j}(t)=v_{i j}(t)-v_{i j}(t-\tau)$. Note that $v_{i j}(t)$ represents the $j^{\text {th }}$ entry of the $i^{\text {th }}$ row of $\boldsymbol{V}(\mathrm{t})$, and $\lambda_{i}(t)$ are the eigenvalues of $\boldsymbol{H}^{\dagger}(t) \boldsymbol{H}(t)$. Furthermore, $Z_{k j}$ is a family of 


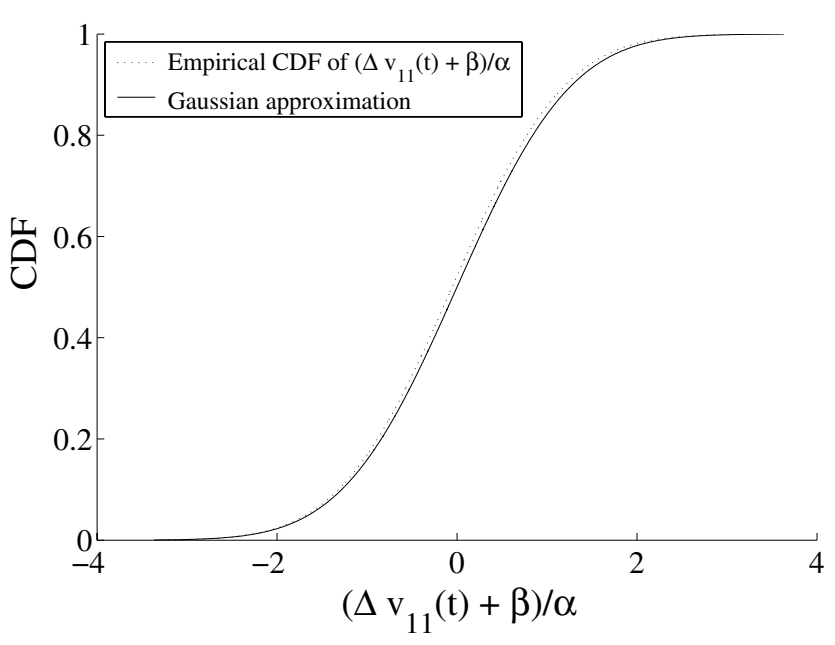

Fig. 1. Numerical verification of the SDE.

complex Gaussian random variables with zero mean and unit variance, independent of each other and of the $\lambda_{i}(t)$ processes.

The eigenvector process has been assumed to be continuous in [13], but due to the non-uniqueness of eigenvectors, continuity of the eigenvector process is not guaranteed during computation. Hence, to deal with this issue, we always rotate the first element of the eigenvectors to a common reference axis (the positive real-axis is employed for convenience), to ensure the continuity. In order to confirm the validity of (7), we write (7) in the form of $\Delta v_{i j}(t)=\alpha Z_{k j}-\beta$. Then, we inspect the empirical distribution of $\left(\Delta v_{i j}(t)+\beta\right) / \alpha$, which should be approximately Gaussian with zero mean and unit magnitude variance. The results in Fig. 1 were obtained from the SDE for $d v_{11}(t)$ in a $(2,2)$ system. As can be seen, the simulated values of $\left(\Delta v_{i j}(t)+\beta\right) / \alpha$ match the Gaussian extremely well. Similar results were obtained for the other eigenvector elements $v_{12}(t)$, $v_{21}(t)$ and $v_{22}(t)$ in the $(2,2)$ case and also for larger systems.

\section{Derivation of the SINR}

In this section, we show how the SDE can be applied to derive the SINR in the presence of feedback delay. If (7) is re-written in matrix notation, we have

$$
\Delta \boldsymbol{V}=\boldsymbol{V}(t)-\boldsymbol{V}(t-\tau) \approx \boldsymbol{V}(t-\tau) \boldsymbol{A}(t-\tau)
$$

In equation (8), some terms have time index $t$ and some have $t-\tau$. In order to simplify notation, in the rest of the paper we denote the eigenvalues at time $t$ by $\lambda_{i}(t)$ and at time $t-\tau$ by $\lambda_{i}$. The elements $a_{i j}$ of $\boldsymbol{A}(t-\tau)$ are given by

$$
a_{i j}= \begin{cases}-\pi^{2} f_{D}{ }^{2} \tau^{2} \sum_{k \neq j} \frac{\lambda_{k}+\lambda_{j}}{\left(\lambda_{k}-\lambda_{j}\right)^{2}}, & i=j \\ \sqrt{2} \pi f_{D} \tau \sqrt{\frac{\lambda_{k}+\lambda_{j}}{\left(\lambda_{k}-\lambda_{j}\right)^{2}}} Z_{k j}, \quad i \neq j .\end{cases}
$$

Rewriting (8), we have $\boldsymbol{V}(t)=\boldsymbol{V}(t-\tau)+\Delta \boldsymbol{V}$. Thus,

$$
\boldsymbol{V}^{\dagger}(t) \approx[\boldsymbol{I}+\boldsymbol{A}(t-\tau)]^{\dagger} \boldsymbol{V}(t-\tau)^{\dagger}
$$

where $\boldsymbol{I}$ is the identity matrix. This then can be substituted into (5), so the overall system equation becomes

$$
\begin{aligned}
\widehat{\boldsymbol{y}}(t) & \approx \boldsymbol{D}(t)[\boldsymbol{I}+\boldsymbol{A}(t-\tau)]^{\dagger} \boldsymbol{V}(t-\tau)^{\dagger} \boldsymbol{V}(t-\tau) \boldsymbol{x}(t)+\widetilde{\boldsymbol{n}}(t) \\
& =\boldsymbol{D}(t)\left[\boldsymbol{I}+\boldsymbol{A}^{\dagger}(t-\tau)\right] \boldsymbol{x}(t)+\widetilde{\boldsymbol{n}}(t) .
\end{aligned}
$$

Therefore, it is easy to show that the received signal on the $i^{\text {th }}$ eigenmode is given by

$$
\widehat{y}_{i}(t) \approx \sqrt{\lambda_{i}(t)}\left[\left(1+a_{i i}^{*}\right) x_{i}(t)+\sum_{j \neq i} a_{j i}^{*} x_{j}(t)\right]+\widetilde{n}_{i}(t) .
$$

As the signal and interference components are now explicitly identified in (11), their power can be derived. In order to simplify the expressions, we assume that all data, $x_{i}$, have $\mathrm{E}\left|x_{i}\right|^{2}=1$. In this situation, the instantaneous desired signal and interference powers, $S_{i}$ and $I_{i}$ respectively, are given by

$$
\begin{aligned}
S_{i} & \approx \lambda_{i}(t) \mathrm{E}\left(\left|1+a_{i i}^{*}\right|^{2}\right) \\
& =\lambda_{i}(t) \mathrm{E}\left(1+a_{i i}+a_{i i}^{*}+\left|a_{i i}\right|^{2}\right) \\
& \approx \lambda_{i}(t)\left[1-2 \pi^{2} f_{D}{ }^{2} \tau^{2} \sum_{k \neq i} \frac{\lambda_{k}+\lambda_{i}}{\left(\lambda_{k}-\lambda_{i}\right)^{2}}\right]
\end{aligned}
$$

and

$$
\begin{aligned}
I_{i} & \approx \lambda_{i}(t) \mathrm{E}\left[\sum_{j \neq i}\left|a_{j i}\right|^{2}\right] \\
& =2 \pi^{2} f_{D}{ }^{2} \tau^{2} \lambda_{i}(t) \sum_{j \neq i} \frac{\lambda_{j}+\lambda_{i}}{\left(\lambda_{j}-\lambda_{i}\right)^{2}},
\end{aligned}
$$

where we have averaged over the signal and noise but conditioned on the channel matrix $\boldsymbol{H}$. Note that we have ignored $\left|a_{i i}\right|^{2}$ in (12) because it involves $\tau^{4}$ and $\tau$ is assumed to be small enough to guarantee that $\left|a_{i i}\right|<<1$. In scenarios where the eigenvalues are close together the size of $\left|a_{i i}\right|$ will increase and smaller values of $\tau$ may need to be assumed. However, simulations indicate that this effect does not cause a problem. Hence, the instantaneous SINR is given by

$$
\operatorname{SINR}_{\mathrm{i}} \approx \frac{\lambda_{\mathrm{i}}(\mathrm{t})\left[1-2 \pi^{2} \mathrm{f}_{\mathrm{D}}{ }^{2} \tau^{2} \sum_{\mathrm{k} \neq \mathrm{i}} \frac{\lambda_{\mathrm{k}}+\lambda_{\mathrm{i}}}{\left(\lambda_{\mathrm{k}}-\lambda_{\mathrm{i}}\right)^{2}}\right]}{\sigma^{2}+2 \pi^{2} \mathrm{f}_{\mathrm{D}}{ }^{2} \tau^{2} \lambda_{\mathrm{i}}(\mathrm{t}) \sum_{\mathrm{j} \neq \mathrm{i}} \frac{\lambda_{\mathrm{j}}+\lambda_{\mathrm{i}}}{\left(\lambda_{\mathrm{j}}-\lambda_{\mathrm{i}}\right)^{2}}} .
$$

Note that the loss in signal power is exactly the same as the induced interference and both are proportional to $\left(f_{D} \tau\right)^{2}$.

To verify these expressions, a set of simulations has been performed. We set a feedback delay of $0.1 \mathrm{~ms}$ and compared our analytical results, from (12) to (14), against simulation data for the signal power, interference power and SINR. Excellent agreement was found in all cases, and the SINR comparison is shown in Fig. 2 for $\mathrm{SINR}_{1}$ in a $(2,2)$ system. In Fig. 2 we simulated 20 independent channel matrices at times $t-\tau$ and $t$. This yields 20 independent SINR values using (14). These analytical SINR values were then verified by simulation in the following way. Fixing the channels at time $t-\tau$ we then generate 1,000 values of the channel at time $t$ using independent Jakes processes and calculate the average SINR by simulation for each of the 20 cases. 


\section{The (2,2) Case}

Some useful conclusions can be drawn from an inspection of the $(2,2)$ case. The signal power loss on the first eigenmode is the same as the interference on the first eigenmode and is equal to $I_{1}=2 \pi^{2} f_{D}^{2} \tau^{2} \lambda_{1}(t)\left(\lambda_{1}+\lambda_{2}\right)\left(\lambda_{1}-\lambda_{2}\right)^{-2}$. On the second eigenmode, we have the the signal power loss $I_{2}=2 \pi^{2} f_{D}^{2} \tau^{2} \lambda_{2}(t)\left(\lambda_{1}+\lambda_{2}\right)\left(\lambda_{1}-\lambda_{2}\right)^{-2}$. Since $\lambda_{1}(t)>$ $\lambda_{2}(t)$, we have $I_{1}>I_{2}$, and typical eigenmode traces over time show many occasions when $\lambda_{1}(t)>>\lambda_{2}(t)$, leading to $I_{1}>>I_{2}$. Although the absolute loss in signal power is greater for the first eigenmode, the relative loss is identical, since $I_{1} / \lambda_{1}(t)=I_{2} / \lambda_{2}(t)$. The assumption in [8] that signal power loss is spread equally over the remaining eigenmodes is not valid here. However, the work in [8] focussed on a more complex scenario where such an approximation might be more reasonable.

The effect of interference on the different eigenmodes is considered in more details later. Inspection of $I_{1}$ and $I_{2}$ shows that the interference is largest when $\lambda_{1}$ and $\lambda_{2}$ are both large and similar in value. In this situation, $\lambda_{1}+\lambda_{2}>>0$ and $\lambda_{1}-\lambda_{2} \approx 0$ leading to large values of $I_{1}$ and $I_{2}$. Note that two large similar eigenvalues leads to high capacity and in such situations, with adaptive SVD, the use of the link would be maximized by employing large constellations. Unfortunately, this scenario is also the one with the highest interference. Hence, caution may be necessary in switching to higher level schemes.

\section{E. The Interference Factor}

From the results in the previous section, we have observed the following: if $f_{D}$ and $\tau$ are assumed to be constant, both the loss in signal power and the magnitude of the interference power are proportional to the value of

$$
Q_{i}=\lambda_{i}(t) \sum_{k \neq i} \frac{\lambda_{k}+\lambda_{i}}{\left(\lambda_{k}-\lambda_{i}\right)^{2}}
$$

In other words, the SINR on the $i^{t h}$ eigenmode degrades more severely with larger values of $Q_{i}$. Hence, this parameter can be identified as an interference factor, and can be used as a metric that measures the sensitivity of system performance to feedback delay. From (15), we can infer that one of the worst scenarios is when two eigenvalues are close to each other. This can be explained using the notion of repelling force, which has been mathematically interpreted from the drift term of the eigenvalue SDE [14] (see Fig. 3). The denominator of the drift term in the SDE demonstrates the phenomenon that eigenvalues tend to repel and move away from each other if they are close together. The whole eigen-structure experiences dramatic change at that time. Thus, rapid changes in the eigenvectors caused by repelling eigenvalues may result in large differences between the true and outdated steering matrices. At such times, the loss in signal power and the induced interference power are particularly significant.
The occurence of large $Q$ values can also be seen from an inspection of the joint density function,

$$
\begin{aligned}
f\left(\lambda_{1}, \ldots, \lambda_{m}\right)= & \prod_{i=1}^{m}[(n-i) !(m-i) !]^{-1} \exp \left(-\sum_{i=1}^{m} \lambda_{i}\right) \\
& \times \prod_{i=1}^{m} \lambda_{i}^{n-m} \prod_{i<j}\left(\lambda_{i}-\lambda_{j}\right)^{2}
\end{aligned}
$$

as given in [1]. Consider the term $\sum_{k \neq i}\left(\lambda_{k}+\lambda_{i}\right)\left(\lambda_{k}-\lambda_{i}\right)^{-2}$ in $Q_{i}$. The mean of this term exists since the denominator cancels with the numerator of $f\left(\lambda_{1}, \ldots, \lambda_{m}\right)$. The variance, however, is infinite since it involves terms of the form $\left(\lambda_{k}-\lambda_{i}\right)^{-2}$ leading to a divergent integral. Hence, $Q_{i}$ has a long tailed distribution and very large values will occur at times.

\section{RESULTS}

In order to illustrate the influence of $Q$, we have carried out simulations of the strongest eigenmode of several MIMO systems, and examined the relationship between the value of $Q$ and the instantaneous error performance with equal-power BPSK and a feedback delay of $0.1 \mathrm{~ms}$. Furthermore, we assume the mobile unit is moving with a speed of $5 \mathrm{Km} / \mathrm{Hr}$ and the system carrier frequency is $5.725 \mathrm{GHz}$ (HyperLan 2 standard), which gives a Doppler frequency of $26.5 \mathrm{~Hz}$. Considering the coherence time corresponding to the Doppler frequency of $26.5 \mathrm{~Hz}$, the feedback delay of $0.1 \mathrm{~ms}$ is very small and will have very little impact on the quality of the channel estimates. However, as discussed earlier, regardless of the feedback delay, the SINR degrades whenever two or more eigenvalues are nearly the same. The results for $(2,2),(2,4)$ and $(4,4)$ systems are shown in Figs. 4, 5 and 6 respectively. The lower curve is the value of $Q$ and the top curves are the instantaneous BER curves (solid line - with feedback delay, dashed line - perfect CSI). Note that, conditioned on $\lambda_{1}, \lambda_{2}, \ldots, \lambda_{m}$, the interference-plus-noise component in (11) is Gaussian due to the $Z_{k j}$ terms. Hence, we have a signal in AWGN with SINR given by (14), and the corresponding instantaneous error probability is simply $\Phi\left(-\sqrt{\text { SINR }_{\mathrm{i}}}\right)$ for BPSK, where $\Phi$ is the cumulative distribution function of a standard Gaussian variable.

From Figs. 4 - 6 we observe that the effect of feedback delay on $\lambda_{1}$ increases as the system size increases. This can be seen from the gap between the BER curves for perfect CSI and delayed CSI. Inspection of Figs. 4-6 also shows the relationship between BER and $Q$. When the BER for delayed CSI peaks at a high value, with a large gap to the perfect CSI case, there is a corresponding peak in the $Q_{1}$ value. Furthermore, the average $Q_{1}$ value increases from $(2,2)$ to $(4,4)$ systems, and this drives the BER curves further apart. This can also be observed from the analytical result (13) since large system sizes have more terms in the interference factor. Fig. 7 shows $Q_{1}$ and $Q_{4}$ over time for a $(4,4)$ system. As discussed above, for the $(2,2)$ case, there is a considerable difference in the interference levels for $\lambda_{1}$ and $\lambda_{4}$ with the largest eigenmode experiencing much higher interference. Fig. 8 shows the percentage power lost to interference $(I)$ 


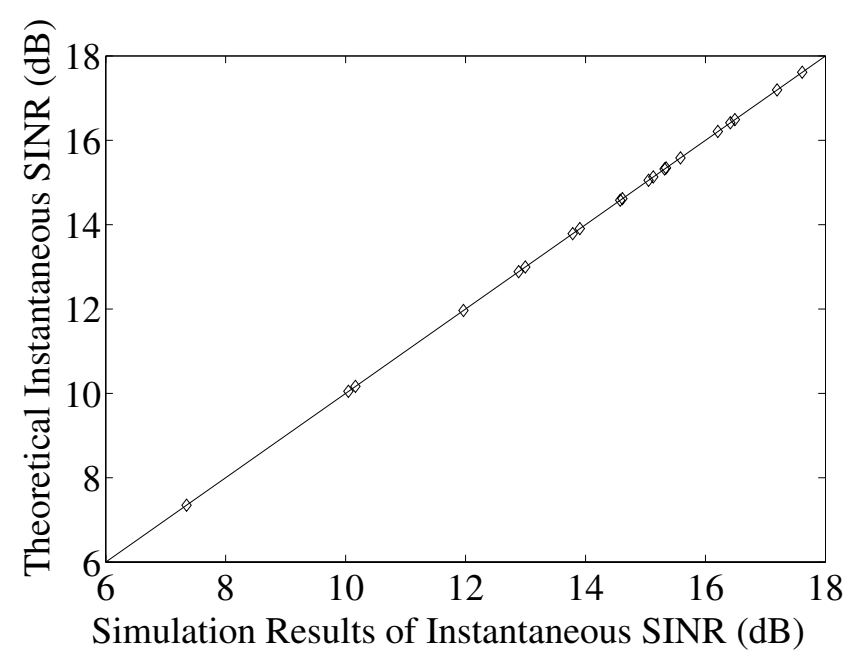

Fig. 2. The comparison between analytical SINR and simulation results.

relative to the original signal power $(\lambda)$. The difference in percentage power losses is less clear, with $\lambda_{4}$ experiencing more periods of heavy relative interference. Fig. 3 shows $\lambda_{1}$, $\lambda_{2}$ and $Q$ for a $(2,2)$ system, where $Q_{1}=Q_{2}=Q$. Clearly seen are the "repelling forces" where $\lambda_{1} \approx \lambda_{2}$ results in a large $Q$ value and a divergence of $\lambda$ values shortly afterwards. Also, note the largest $Q$ peaks are caused by $\lambda_{1} \approx \lambda_{2}$ and $\lambda_{1}, \lambda_{2}$ significantly larger than zero. Hence, in high capacity scenarios, there can be very large interference. Additionally, the long tailed nature of $Q$ is clear, with a majority of small to moderate values and occasional very large peaks. Finally in Fig. 9 we take a global look at error performance, averaging over the $\lambda$ values to get average BER results for $\lambda_{1}, \lambda_{2}, \lambda_{3}$ and $\lambda_{4}$ in a $(4,4)$ system. As is well-known [7] the time delay leads to a floor in BER performance which is most noticeable for $\lambda_{1}$.

\section{CONClusion}

In this paper, we have derived novel analytical expressions for the instantaneous signal power, self-interference power and hence the SINR of MIMO eigenmode transmission with feedback delay. These results have been verified through simulations. From these expressions, it can be shown that the loss in signal power is identical to the induced interference. Also, this power is proportional to a single number which can be determined using the channel eigenvalues. Thus, we propose this parameter, $Q$, as a novel channel metric that can be employed to gauge the performance sensitivity to feedback delay. Using the analytical results we can see an increase in sensitivity to delay as the system size increases and large differences between absolute interference levels in the different eigenmodes. However, the relative interference on the eigenmodes tends to be more similar. Also, the interference is a long tailed variable which can produce large values often at times of high capacity when the link would ideally be operating at high rates.

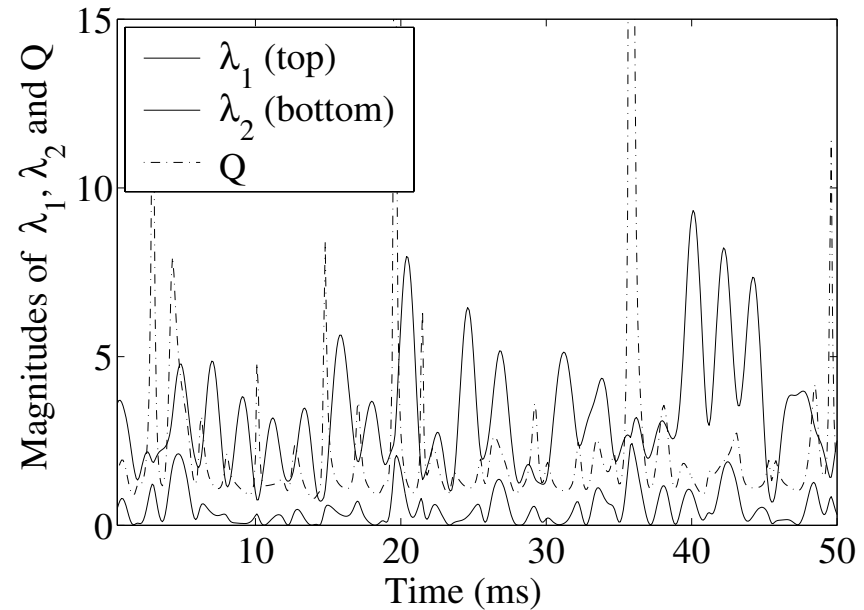

Fig. 3. The comparison between instantaneous eigenvalues and $Q$ value in a $(2,2)$ system.

\section{REFERENCES}

[1] I. E. Telatar, "Capacity of multi-antenna Gaussian channels," European Trans. on Telecomm. Related Technol., vol. 10, pp. 585-595, Nov. 1999.

[2] J. B. Andersen, "Array gain and capacity for known random channels with multiple element arrays at both ends," IEEE J. Select. Areas Commun., vol. 18, pp. 2172-2178, Nov. 2000.

[3] G. Lebrun, J. Gao, and M. Faulkner, "MIMO transmission over a timevarying channel using SVD," IEEE Trans. Wireless Commun., vol. 4, pp. 757-764, Mar. 2005.

[4] K. Miyashita, T. Nishimura, T. Ohgane, Y. Ogawa, Y. Takatori, and K. Cho, "High data-rate transmission with eigenbeam-space division multiplexing (E-SDM) in a MIMO channel," in Proc. IEEE VTC Fall, 2002, pp. 1302-1306.

[5] D. L. Goeckel, "Adaptive coding for time-varying channels using outdated fading estimates," IEEE Trans. Commun., vol. 47, pp. 844855, June 1999.

[6] V. T. Ermolayev, A. G. Flaksman, I. P. Kovalyov, and I. M. Averin, "Weight error loss in MIMO systems with adaptive transmit and receive beamformers," in Proc. IEEE Int. Conf. Ant. Theo. and Tech., Sept. 2003, pp. $333-336$.

[7] A. Cano-Gutierrez, M. Stojanovic, and J. Vidal, "Effect of channel estimation error on the performance of SVD-based MIMO communication systems," in Proc. IEEE PIMRC, 2004, pp. 508-512.

[8] T. S. Ho, K. Sakaguchi, and K. Araki, "Performance analysis of MIMO eigenmode transmission system under realistic channel and system conditions," in Proc. IEEE VTC Spring, 2004, pp. 708-712.

[9] E. N. Onggosanusi, A. Gatherer, A. G. Dabak, and S. Hosur, "Performance analysis of closed-loop transmit diversity in the presence of feedback delay," IEEE Trans. Commun., vol. 49, pp. 1618-1630, Sept. 2001.

[10] S. Spiteri, G. Lebrun, and M. Faulkner, "Prediction for time-varying SVD systems," in Proc. IEEE PIMRC, 2004, pp. 1608-1612.

[11] G. Lebrun, S. Spiteri, and M. Faulkner, "Channel estimation for an SVD-MIMO system," in Proc. IEEE ICC, 2004, pp. 3025-3029.

[12] T. J. Willink, "MIMO OFDM for broadband fixed wireless access," IEE Proc. Commun., vol. 152, pp. 75-81, Feb. 2005.

[13] M. F. Bru, "Diffusions of perturbed principal component analysis," $J$. Multivariate Anal., vol. 29, pp. 127-136, 1989.

[14] C. Donati-Martin, Y. Doumerc, H. Matsumoto, and M. Yor, "Some properties of the Wishart processes and a matrix extension of the Hartman-Watson laws," Publ. Res. Inst. Math. Sci., vol. 40, pp. 13851412, 2004.

[15] P. J. Smith, P.-H. Kuo, and L. M. Garth, "Level crossing rate for MIMO channel eigenvalues: implications for adaptive systems," in Proc. IEEE ICC, May 2005, pp. 2442-2446. 


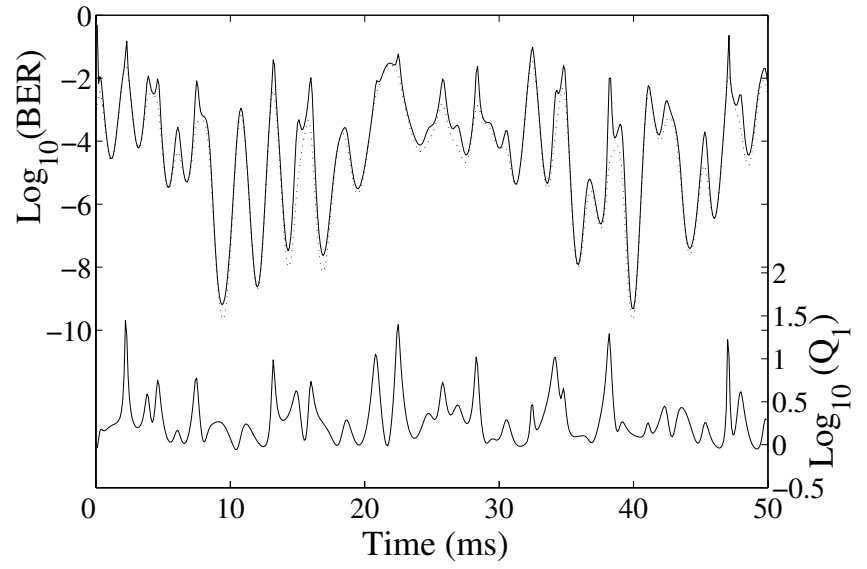

Fig. 4. The comparison between instantaneous error performance with and without feedback delay in a $(2,2)$ system. Also shown is the interference factor $Q_{1}$

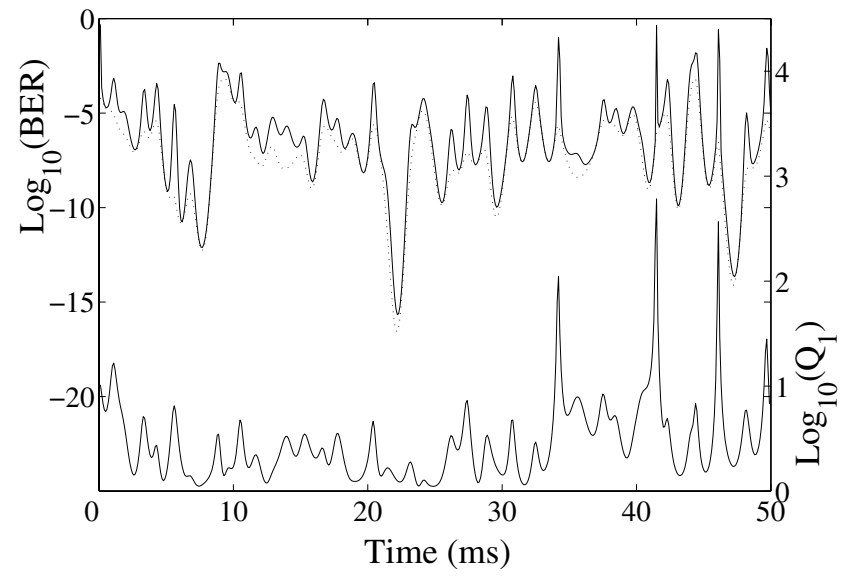

Fig. 5. The comparison between instantaneous error performance with and without feedback delay in a $(2,4)$ system. Also shown is the interference factor $Q_{1}$

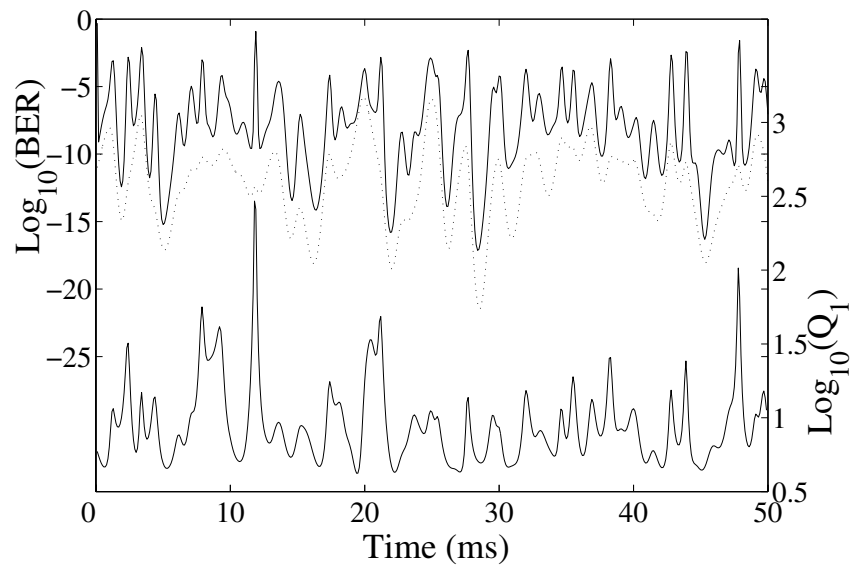

Fig. 6. The comparison between instantaneous error performance with and without feedback delay in a $(4,4)$ system. Also shown is the interference factor $Q_{1}$

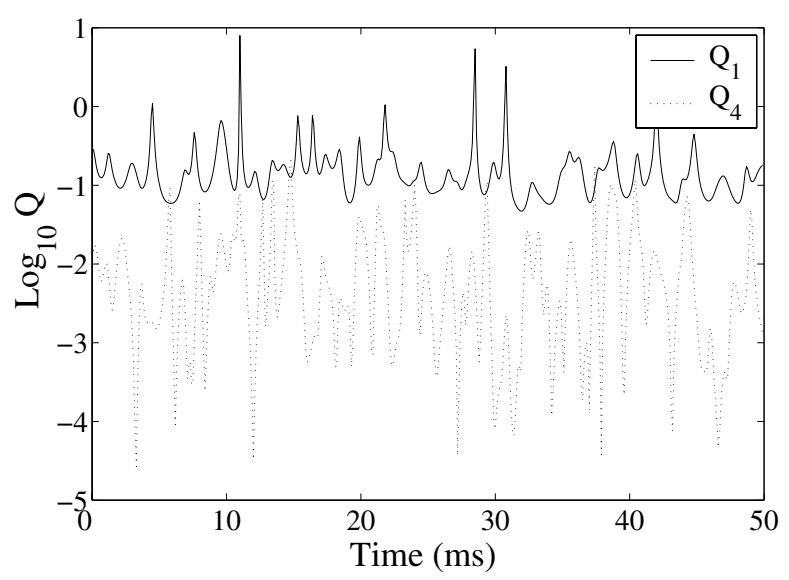

Fig. 7. The instantaneous comparison between $Q_{1}$ and $Q_{4}$ in a $(4,4)$ system.

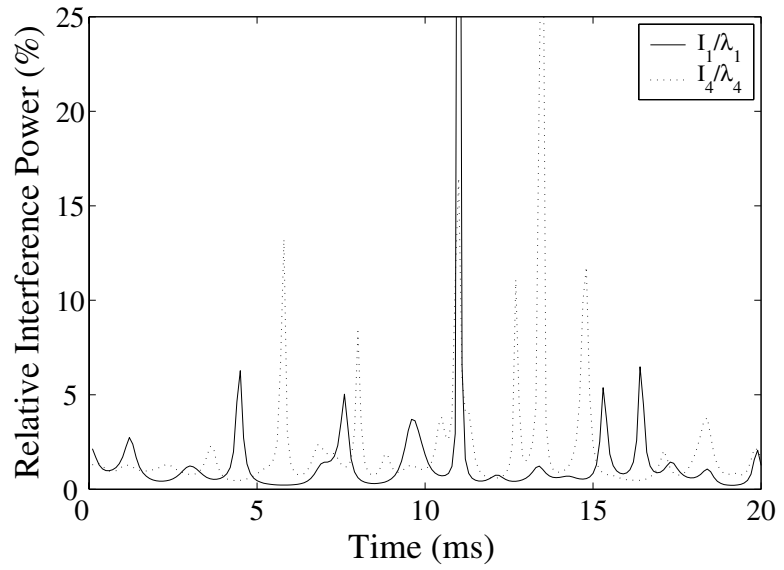

Fig. 8. The relative power loss of the strongest and weakest eigenmodes of a $(4,4)$ system.

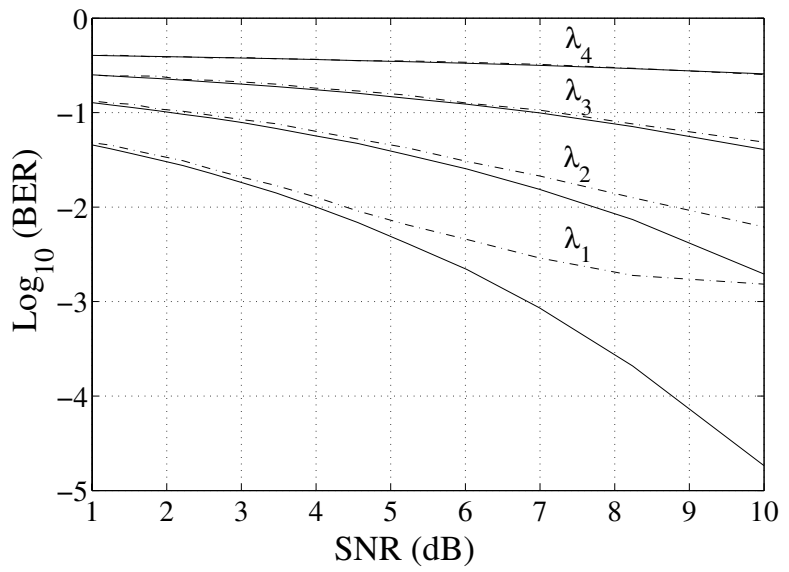

Fig. 9. Average error probability curve of a $(4,4)$ system. Solid lines and dashed lines represent the behavior without and with feedback delay respectively. 